\title{
BRINGING DISCOVERY OF LOW-PROFILE SIGHTSEEING SPOTS FOR INBOUND TOURISTS - ACQUIRING TOURING SERENDIPITY USING A JOINT TOPIC MODEL -
}

\author{
Satona Shirai, Takayasu Yamaguchi and Hiroshi Uehara \\ Akita Prefectural University \\ 84-4 Aza Ebinokuchi Tsuchiya, Yurihonjo, Akita, Japan
}

\begin{abstract}
This study proposes to provide foreign tourists with various information concerning sightseeing spots, including unvisited ones, in an interpretable manner according to relevance to their preferences. Recently, enormous amounts of travel reviews posted on world travel websites have become an important resource for travelers. Nevertheless, several sightseeing spots potentially attractive to foreigners are left undiscovered because of the information gap between reviews written by foreigners and those written by native tourists. Although the reviews written by native tourists include a wide variety of information that are unknown to foreigners, the information is not conveyed to foreigners efficiently, resulting in inbound tourism within a limited range of standard spots. In this study, a topic model, latent Dirichlet allocation (LDA), is applied to specify relevant reviews for foreigners from miscellaneous topics in the native reviews. Subsequently, the joint topic model, an extended LDA model, is applied to augmented reviews, including reviews written by foreigners and the native reviews extracted by LDA, and acquires categories of recommendable sightseeing spots as a form of topic. Each topic consists of sightseeing spots and their interpretable information, reflecting the interests of the travelers. The proposal was applied to reviews related to sightseeing spots in Japan, and it successfully acquired topics including a broader range of sightseeing spots than using information based only on foreign reviews. Moreover, several spots rarely known to foreigners were found to comply with their preferences, further supporting the performance of the proposal.
\end{abstract}

\section{KEYWORDS}

Social Media, Data Mining, Data Analytics, Recommendation, Tourism, Machine Learning

\section{INTRODUCTION}

Promoting inbound tourism via tourist attractions is a common task shared by any country's tourism industry. In many cases, a large portion of the information concerning sightseeing spots is left undiscovered by foreign tourists, limiting the development of inbound tourism. Recently, world travel websites have collected numerous travelers' reviews that have become a useful information source for foreign tourists. Nevertheless, the reviews provide limited variation in visited sightseeing spots because a large information gap exists between the reviews written by resident tourists and those written by foreign tourists. Although reviews written by resident tourists include much more information than those of foreigners, the information is partitioned by nationality and the information written by resident tourists is not conveyed to foreign tourists. The issue does not come from the difference in language but the difference in their preferences. Foreigners have difficulties in finding information from miscellaneous reviews by native tourists reflecting diverse preferences. Thus, various places familiar to resident tourists are left undiscovered by foreign visitors, even though they are potentially attractive to them.

This paper proposes a method to fill this information gap between resident tourists and foreign tourists in the reviews of a world travel website and enables the recommendation of various sightseeing spots, including lesser-known ones, for foreign tourists. Applying our proposal to Japanese sightseeing spots, we aim to contribute to the revitalization of untapped tourism resources by providing serendipitous recommendations to foreign visitors interested in Japan. 
In Section 2, previous studies on tourism are reviewed, and the issue this study is trying to uncover is presented. In Section 3, the source of the data is introduced. In Section 4, our proposal is elaborated. Subsequently, the results and the evaluation are described, followed by the conclusion.

\section{RELATED WORK}

Among the studies on the recommendation of sightseeing spots, collaborative filtering is one of the major methods for detecting spots to be recommended (Nilashi, 2015; Zhang, 2015; Huang, 2012; Jia, 2016). Nilashi et al. (2015) proposed a recommendation for hotels based on user-based collaborative filtering. Zhang and Wang (2015) also used user-based collaborative filtering based on users' check-in history recorded in social networking services (SNSs). Similarly, Huang and Gartner (2012) adopted GPS that represents the users' trajectory as the data for collaborative filtering. User-based collaborative filtering creates the recommended information based on the similarity among users' historical activities without any semantic validity to support it.

In this respect, several studies applied a topic model, which provides interpretable users' preferences and enables the evaluation of the relevance of recommendation as a form of a topic ( $\mathrm{Hu}, 2013$; Kurashima, 2013; Jiang, 2015). Kurashima et al. (2013) proposed traveler recommendations to unfamiliar sightseeing spots in foreign countries based on their domestic preferences detected by the topic model. In this study, spots are recommended categorically, such as hotels, tower monuments, and museums. Jiang et al. (2015) proposed user-based collaborative filtering combined with topic modeling; the proposal detects users' similarities based on geotags attached to their posts in SNSs. In addition, reviews on the spots indexed by geotags are used to interpret tourists' interests as a form of topic. According to this study, this proposal does not apply to the case of geolocations with sparse reviews.

The sparseness of data has been a common issue for recommendation regardless of the applied method. The cold start problem is well-known in the case of collaborative filtering (Zheng, 2016). The problem pointed out by Jiang et al. (2015) is another aspect of the sparseness in the case of a topic model. Sparse data tend to be overwhelmed by the data occupying most of the users' preferences and result in topics representing very standard preferences without any surprise.

This study applies a joint topic model (JTM)(Mimno, 2009) to recommend sightseeing spots with interpretable information, which enables the evaluation of the relevance of the recommendations. We propose to implement a procedure for augmenting data, thereby improving the sparseness. Applying the augmenting procedure to foreign tourists' reviews concerning Japanese sightseeing spots, we try to provide serendipitous recommendations that tend to be overwhelmed in conventional topic model.

\section{DATA}

In this study, traveler reviews in Trip Advisor, the world's largest travel website, are used. This service covers travel information from 49 countries worldwide and includes an enormous amount of traveler reviews, over 800 million. Despite the volume, the reviews posted by resident travelers tend to include diversities of touring information, while reviews posted by foreign travelers reflect a narrower range of touring information. In this study, the reviews concerning Japanese sightseeing spots are extracted to apply our proposal. The number of reviews extracted by Japanese tourists was 164,570, while that by foreigners was 57,782. Each review from Trip Advisor was annotated by a sightseeing spot of the reviewer's interest. The number of sightseeing spots annotated to Japanese reviews was 492 , whereas the number annotated to foreigners was 233 , which is significantly lower.

A topic model, latent Dirichlet allocation (LDA) (Blei $\mathrm{Ng}$, and Jordan, 2003), was used to analyze the information gap between Japanese and foreigner reviews. LDA classifies words into topics, each of which is represented as a probabilistic distribution of words (called 'topic vector' hereafter). Words with high probability within each topic vector represent the meaning of each topic.

The two boxes in Figure 1 show the results of LDA. The left-hand side represents the topics detected in foreigner reviews, while the right-hand box represents the Japanese ones. Each row in the boxes denotes a topic comprising of two columns: topic No. and top 20 words with a high probability distribution. Some of the topics 
share similar interests (i.e., a similar distribution of topic vectors) between foreigners and Japanese, indicated by the lines connecting the corresponding topics. The number of related topics which share similar interests was 10 out of 70 , implying that the overall topic interests differ between them.

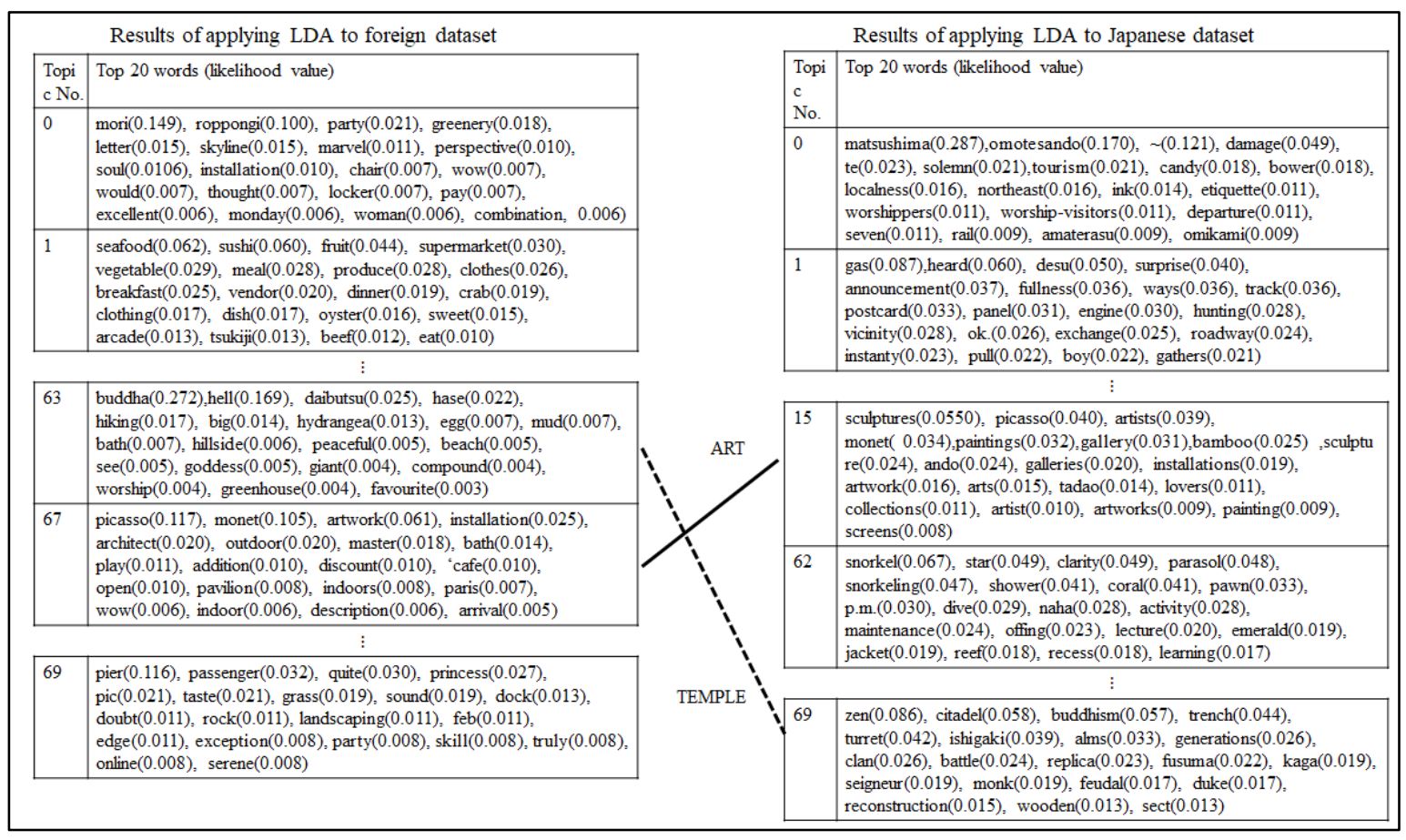

Figure 1. Comparison of the topics of interest between Japanese tourists and foreigners

\section{PROPOSAL}

This study applies a JTM to the reviews to provide serendipitous recommendations in accordance with foreign visitors' preferences. A JTM is an extended LDA which classifies complementary information to the main documents (i.e., reviews) into topics, together with the topic classification of words by conventional LDA. In this study, we use sightseeing spots attached to reviews as complementary information. Based on these datasets, a JTM creates two kinds of topic vectors: 1) a topic vector represented by the word distribution in the reviews, and 2) a topic vector represented by the distribution of sightseeing spots. The semantic similarity of sightseeing spots within each topic vector can be interpreted by the corresponding topic vector represented by the word distribution in the reviews. A correspondence topic model(CTM) (Blei, and Jordan, 2003) which is an extended LDA could be an alternative to a JTM. However, a CTM estimates each topic vector of a sightseeing spot(i.e.,2) above) under the restriction of possible topics assigned to the corresponding main document. Meanwhile, an estimation of a topic by a JTM is not subject to such a restriction. In our proposal, both the topic vectors 1) and 2) described above should be acquired based on all the information across sightseeing spots and reviews. In this respect, a JTM is more suitable than a CTM for our proposal.

Because reviews of Japanese tourists include a much wider variety of sightseeing spots than those of foreign tourists as described in Section 3, translating Japanese reviews into a relevant language might be a way to augment the reviews of foreigners. Nevertheless, this augmentation brings strong bias to the topic classification, compared to the case when only foreigner reviews are considered and results in topics that do not reflect foreigner preferences. Considering this issue, we specify the Japanese reviews coincident with foreigner preferences and extract them for the augmentation.

Figure 2 illustrates the proposed approach. The procedure comprises of two steps. The first one is for detecting corresponding topics between the Japanese and foreigner reviews. The second one is the topic classification for acquiring the recommended sightseeing spots with the semantic interpretation for each 
recommendation. Both steps implement LDAs, a conventional LDA for the first step, and a JTM for the second step. The conventional LDA is used for filtering out reviews reflecting preferences unrelated to foreigners. Once the corresponding topic vectors are detected between Japanese and foreigner reviews, the documents (i.e., reviews) belonging to the topics can be specified from miscellaneous Japanese reviews. The LDA represents each document (i.e., a review) as a distribution of topics. The topic indicating the highest probabilistic value in the distribution is the topic to which each review presumably belongs. Collaborative filtering is not applicable in this case because it relies only on document classification (i.e., reviews) and provides no topic information that enables comparing the corresponding preferences between different documents. In the following, each step is elaborated.

Step 1:

a) applying the conventional LDA to Japanese and foreigner reviews separately,

b) manually specifying the corresponding topics representing similar preferences between Japanese and foreigners, and extracting Japanese reviews that belong to the topics detecting their similarity as mentioned above, and

c) augmenting foreigner reviews by mixing with Japanese reviews.

Step 2: applying JTM to the augmented reviews and acquiring the recommended spots and the semantics for each recommendation.

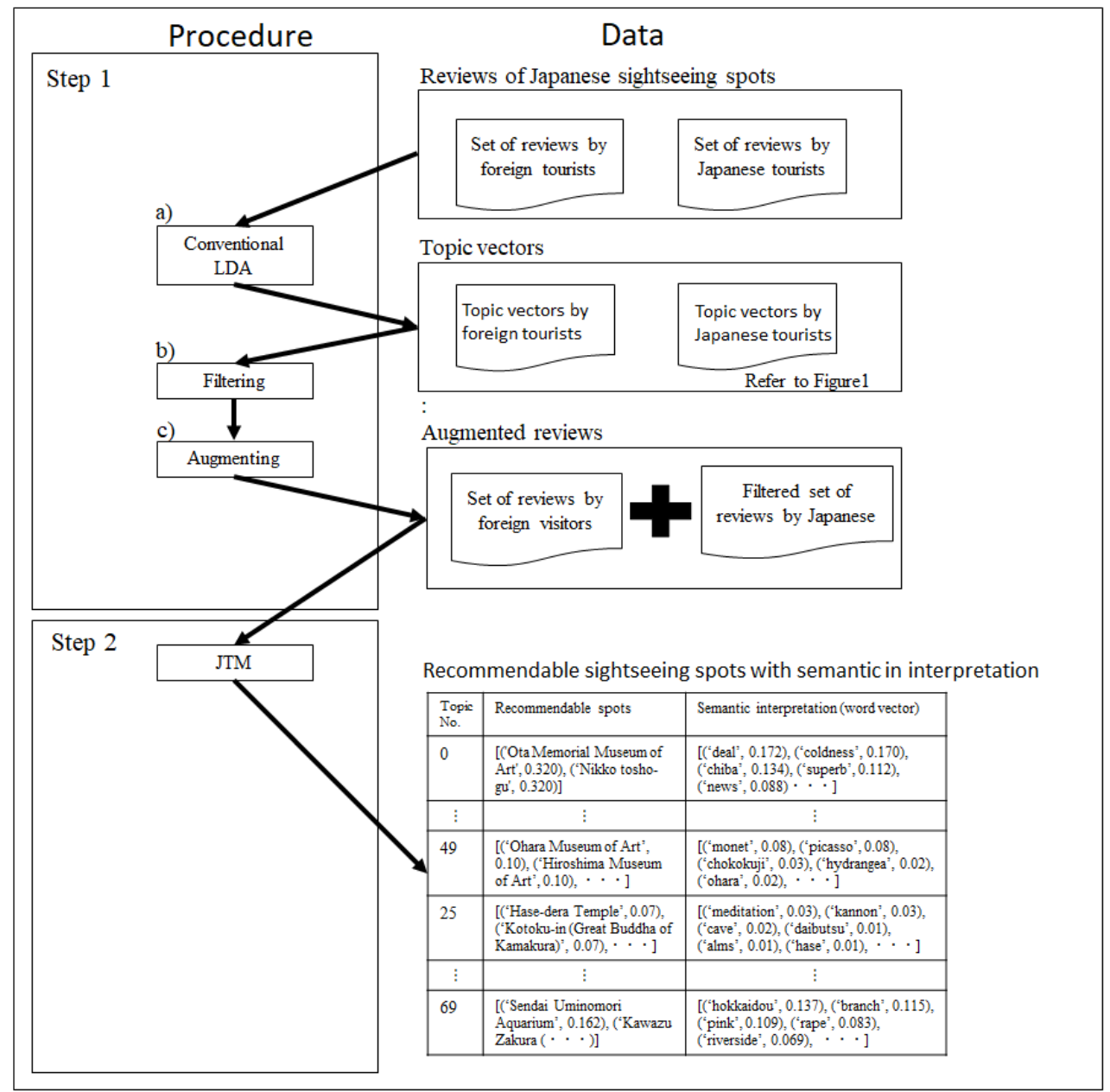

Figure 2. Procedure for creating the augmented reviews and acquiring the recommended information 


\section{RESULT AND DISCUSSION}

Two datasets were prepared for comparative evaluation. One is the foreigner reviews without augmentation, and the other is with the augmentation introduced in Section 4. As a result of applying a JTM to both datasets, the number of sightseeing spots detected from the augmented reviews was 103 , increasing by 24 from only the foreigner reviews.

Table 1 shows two sampled pairs of the corresponding topics acquired from the augmented dataset and the dataset of foreigner reviews only. The shaded rows represent the topics from the augmented reviews, whereas the rows beneath them represent the corresponding topics detected from foreigner reviews. The first pair, corresponding to the first and second rows, represents reviewer interests in arts, which are inferred from the top 20 words with high probability in both of the topic vectors described in the last column. The meanings of these words are similar between the pair, indicating that a JTM successfully detects the topic representing foreigner preferences of interests in the augmented reviews. The third column shows the corresponding sightseeing spots. It appears the number of sightseeing spots is much higher in the first row than in the second one, which supports the effect of the augmentation.

Similarly, both of the third and fourth rows represent the reviewers' interests in temples, and this pair also implies the effect of a JTM with augmented reviews

In addition, several spots from the augmented reviews were found to be popular among Japanese tourists but not among foreign tourists. For example, 'Engaku-ji Temple' and the 'Chishaku-in' in the third row are lesser-known to foreign visitors, reviewed by only a few foreign visitors. Meanwhile, they are popular among Japanese tourists because they not only have a traditional Japanese touch but also a cozy atmosphere. As such, a JTM with augmented reviews could provide serendipitous recommendations for foreign visitors.

As described in Step 1 b) of section 4, the augmented reviews are constructed based on manual interpretation of both the topics from foreigners' reviews and Japanese ones. The manual interpretation is not feasible in case the numerous topics are addressed. One idea for automatic construction is to compute the similarities of topic vectors between foreigners and Japanese. In order to enable the computation, a large thesaurus needs to be prepared, because vocabularies are considerably varied to express similar meaning among the reviews of foreigners and Japanese. The automated augmentation is left in our future work.

Table 1. Samples of the results of recommendable sightseeing spots related to the topic

\begin{tabular}{|c|c|c|c|}
\hline Data & $\begin{array}{l}\text { Topic } \\
\text { No. }\end{array}$ & Recommended sightseeing spots (likelihood value) & $\begin{array}{l}\text { Semantic interpretation } \\
\text { (Top } 20 \text { words inferred by JTM) }\end{array}$ \\
\hline $\begin{array}{l}\text { Augmented } \\
\text { reviews }\end{array}$ & 49 & $\begin{array}{l}\text { Ohara Museum of Art }(0.10) \text {, Hiroshima Museum of Art }(0.10) \text {, Ota } \\
\text { Memorial Museum of } \operatorname{Art}(0.10) \text {, The Hakone Open-Air Museum(0.10), } \\
\text { Pola Museum of Art }(0.10) \text {, Chihiro Art Museum Azumino( }(0.10) \text {, Hiroshi } \\
\text { Senju Museum Karuizawa( }(0.08) \text {, Mother Farm }(0.08) \text {, Katsura Imperial } \\
\text { Villa(0.06), Tokyo Sea Life Park( }(0.02)\end{array}$ & $\begin{array}{l}\text { monet, picasso, chokokuji, hydrangea, ohara, pain } \\
\text { ter, mercy, print, annex, gogh, renoir, mercy-sama, } \\
\text { chagall, van, impressionism, impressionist, writer, } \\
\text { outdoor, munakata, gora }\end{array}$ \\
\hline $\begin{array}{l}\text { Foreigner's } \\
\text { reviews }\end{array}$ & 67 & $\begin{array}{l}\text { The Hakone Open-Air Museum( } 0.33) \text {, Chichu Art Museum( } 0.30) \text {, Pola } \\
\text { Museum of Art }(0.12) \text {, Ohara Museum of Art }(0.09) \text {, Hiroshima Museum of } \\
\text { Art }(0.09)\end{array}$ & $\begin{array}{l}\text { picasso, monet, artwork, installation, architect, } \\
\text { outdoor, master, bath, play, addition, discount, cafe, } \\
\text { open, pavilion, indoors, paris, wow, indoor, } \\
\text { description, arrival }\end{array}$ \\
\hline $\begin{array}{l}\text { Augmented } \\
\text { reviews }\end{array}$ & 25 & $\begin{array}{l}\text { Hase-dera Temple }(0.07) \text {, Kotoku-in Great Buddha of Kamakura)(0.07), } \\
\text { Engaku-ji Temple }(0.07) \text {, Daihonzan Eihei-ji Temple }(0.07) \text {, Memorial Ship } \\
\text { MIKASA }(0.07) \text {, Chishaku-in( }(0.07) \text {, Horyu-ji Temple }(0.07) \text {, Toshodai-ji } \\
\text { Temple }(0.07) \text {, Aokigahara Forest }(0.05) \text {, Enoshima Island( }(0.05) \text {, Kinkakuji } \\
\text { Temple }(0.044) \text {, Ninna-ji Temple }(0.044) \text {, Toji }(0.044) \text {, Omiwa Shrine }(0.029) \text {, } \\
\text { Atsuta Jingu Shrine( }(0.014) \text {, Okayama Castle }(0.014) \text {, Nigatsu-do } \\
\text { Temple( }(0.014) \text {, Goshikinuma Pond }(0.014)\end{array}$ & $\begin{array}{l}\text { meditation, kannon, cave, daibutsu, alms, hase, } \\
\text { kitakamakura, kita-kamakura, monzen, sanctum, } \\
\text { sutra, verdure, pile, incense, } \\
\text { mikado, virtue, fragrance, lichen, tomb, monk }\end{array}$ \\
\hline $\begin{array}{l}\text { Foreigner's } \\
\text { reviews }\end{array}$ & 63 & $\begin{array}{l}\text { Hase-dera Temple( } 0.27) \text {, Kotoku-in Great Buddha of Kamakura(0.24), } \\
\text { Hells of Beppu( }(0.22) \text {, Kamakura Hiking Trails }(0.07) \text {, Nanzoin }(0.07) \text {, } \\
\text { Tochoji Temple }(0.07)\end{array}$ & $\begin{array}{l}\text { buddha, hell, daibutsu, hase, hiking, big, } \\
\text { hydrangea, egg, mud, bath, hillside, } \\
\text { peaceful, beach, see, goddess, giant, compound, } \\
\text { worship, greenhouse, favourite }\end{array}$ \\
\hline
\end{tabular}




\section{CONCLUSION}

This paper proposed to provide a wider range of sightseeing spots for foreign tourists, including unvisited ones, in an interpretable manner with respect to which spots are suitable for their preferences. Despite plenty of reviews on world travel websites, the information on sightseeing spots has been circulating among domestic tourists and leaving foreigners uninformed despite their potential interest. To overcome this issue, we specified domestic reviews related to foreigner preferences and filtered out unrelated ones by adopting LDA to augment adequate information for foreigners. Applying a JTM to the augmented reviews provided a wider range of recommendable sightseeing spots, including lesser-known candidates to foreigners, along with interpretable background information concerning preferences.

Although this study only examined data related to Japan, this approach is expected to be effectively applicable for disseminating inbound tourism information in other countries. Future work will apply the proposed approach to other countries and evaluate its performance.

\section{REFERENCES}

Blei, D.M., Ng, A.Y. and Jordan, M.I., 2003. Latent dirichlet allocation. Journal of Machine Learning Research, Vol. 3 , No., pp. 993-1022.

Blei, D.M., Jordan, M.I., 2003. Modeling annotated data. Proceedings of the 26th annual international conference on Research and development in informaion retrieval, pp. 127-134.

Jiang, S., Qian, X., Shen, J., Fu, Y. and Mei, T., 2015. Author topic model-based collaborative filtering for personalized POI recommendations. IEEE Transactions on Multimedia, Vol. 17, No. 6, pp. 907-918.

Kurashima, T., Iwata, T., Hoshide, T., Takaya, N. and Fujimura, K., 2013. Geo topic model: joint modeling of user's activity area and interests for location recommendation. In Proceedings of the sixth ACM international conference on Web search and data mining. Rome, Italy, pp. 37-384.

Mimno, D., Wallach, H., Naradowsky, J., Smith, D.A. and McCallum, A., 2009. Polylingual topic models. In Proceedings of the 2009 conference on empirical methods in natural language processing. Pennsylvania, USA, pp. 880-889.

Hu, B. and Ester, M., 2013. Spatial topic modeling in online social media for location recommendation. In Proceedings of the 7th ACM conference on Recommender systems. Hong Kong, China, pp. 25-32.

Huang, H. and Gartner, G., 2014. Using trajectories for collaborative filtering-based POI recommendation. International Journal of Data Mining, Modelling and Management, Vol. 6, No. 4, pp. 333-346.

Zhang, C. and Wang, K., 2016. POI recommendation through cross-region collaborative filtering. Knowledge and Information Systems, Vol. 46, No. 2, pp. 369-387.

Nilashi, M., bin Ibrahim, O., Ithnin, N. and Sarmin, N.H., 2015. A multi-criteria collaborative filtering recommender system for the tourism domain using Expectation Maximization (EM) and PCA-ANFIS. Electronic Commerce Research and Applications, Vol. 14, No. 6, pp. 542-562.

Jia, Z., Yang, Y., Gao, W. and Chen, X., 2015, February. User-based collaborative filtering for tourist attraction recommendations. In 2015 IEEE International Conference on Computational Intelligence \& Communication Technology. City, Country, pp. 22-25. 